\title{
Experimental Evaluation of Internet of Things in the Educational Environment
}

\author{
https://doi.org/10.3991/ijep.v7i3.7187 \\ Amr Elsaadany $\left(^{\bowtie}\right)$, Mohamed Soliman \\ Pharos University in Alexandria \\ amr.sadany@pua.edu.eg
}

\begin{abstract}
In the trials of utilization of technology for the society, efforts have shown benefits of the ICT use in facilitating education from different perspectives based on different waves of technological change. The recent development in technologies has also resulted in change of user behavior and usage patterns towards different areas of life, and consequently in the area of education. A new wave of change has started and is expecting to proliferate with stronger connectivity and interoperability of various devices, named as the Internet of Things (IoT). The internet of things is expected to give strong impacts on different areas of life including healthcare, transportation, smart homes, smart campus, and more. Consequently, there are inherent benefits to the education environment that are not yet well established in literature. The paper studies the potential benefit and impact of the IoT evolution concept in both the physical and the virtual learning environment and suggests a paradigm with use case scenarios. The results of an experimental evaluation on the aspects of applying IoT technology in education are presented and discussed in order to verify the set of related hypotheses.
\end{abstract}

Keywords-Internet of things, technology enhanced learning, smart campus, smart city

\section{Introduction}

The recent development in device connectivity and interoperability opened the way for the development of the Internet of Things (IoT), [1]. The internet of things is expected to impact various areas of life including smart homes, smart campus, and more. Its usage for scenarios in the smart city paradigm is apparent where many applications are becoming under fast development, notably in the e-health market [2]. Consequently, there are inherent benefits to the education environment. An apparent example is the physical usage in the smart space or the concept of the smart campus.

This paper aims to answer questions like how does the IoT technology and its applications affect the education environment. This can be done by investigating how IoT can transform the educational environment and make it more application-centric, with the introduction of new educational services such as automated attendance or other e-learning activities. In other words, there is a need to investigate how IoT con- 
nected devices can change the daily student behavior and the evolution of new use case scenarios.

In order to measure the effect of the IoT technology on education, we need to know the students' awareness of this technology and their perspective view on its future applicability. Thus, we try to assess the level of knowledge of the students regarding this technology and its applications in various engineering disciplines and the effects on smart cities way of life, including manufacturing, transportation, and healthcare. It is also necessary to assess the importance of teaching IoT technology into undergraduate engineering level and as such, we evaluate the flexibility that IoT give in conducting the learning activities and accessing the learning resources, and finally, measure the importance of IoT course during early levels of engineering education.

This paper is organized as follows: section 2 gives background and motivation which covers the requirements for an IoT enabled campus and the different perspectives upon the IoT paradigm shift. Section 3 provides the evaluation experiment setup and the results analysis. Section 4 gives the conclusion of the paper.

\section{Background and Motivation}

It is important to study the potential benefit and impact of the IoT evolution concept in both the physical and the virtual learning environment. It is equally important to analyze the usage of the internet of things in virtual educational environments and e-learning systems in a smart campus [3]. Furthermore, the usage in the smart city paradigm is apparent where increased connectivity and interoperability increase the services given to the citizen.

Increased connectivity and smartness of the devices, with the orientation to give services to the learner, can increase the value addition and smartness to the educational environment. Given recent research in this area, new applications are surfacing day after day. Common to the applications, is the targeting of the new users who are interested in utilizing the technology for education, as these users are digital natives by definition [4]. Yet it is to discover and build on the new paradigm for the educational purposes.

Given the characteristics of the paradigm, the user will become able to obtain justin-time information about a constellation of devices which are able to have machineto-machine (M2M) conversations among each other and with other user devices (such as smart watch or smartphone). This work attempts to discover details of such interactions in scenarios that apply to the physical smart campus.

Using the IoT to facilitate the educational environment for student, faculty members, and other staff members yields potential new learning services and scenarios. Examples of the service to be provided include:

- Smart classroom; which is equipped with the IoT controlled objects to give the ability for remote functions. These functions include the ability to set the class room ahead of lecture time. The instructor can set the overhead projector, set the lighting, and set the room temperature, allowing for time and energy saving. The 
equipment to be controlled must be able to receive orders to start a certain action as such they must be equipped with sensors.

- Smart parking areas; which is equipped with sensors to control the occupancy of the parking spaces and gives the indications about the available capacity of the area, again saving time and energy [5].

- Smart secure environment; which is equipped with camera sensors that provides signals to a security control room to increase campus security and avoid unwanted incidents [6].

- Smart student feedback; which allows students to participate in educational environment improvement through a sensing mobile application [6].

To achieve a smart educational environment, there is a need for IoT infrastructure to be in place; composed of sensing devices, communication links, and user applications (typically, using cloud computing). The beauty of this infrastructure is that it can be introduced gradually; adding smartness as it grows, and not requiring major capital investment initially. An organization can build the smartness step by step, assessing the output of the system as it grows. The reports to be generated by the system applications can be used to indicate which area of the infrastructure have precedence over others.

There is no doubt of the recent proliferation of social networks and its impact on user behavior and technology usage patterns. This, combined with the IoT being social itself can bring up new scenarios to the benefit of increasing user engagement [7], especially in learning with different scenarios. It is thus believed that the areas of the Social Internet of Things (SIoT) with smart objects can add to such interest for promoting education in the real and virtual educational environment. For example, social collaborative filtering in educational recommender systems [8] increases the attention and trust to engage in a particular educational activity while being able to get recommendations and support from the user social network on the spot.

Linked to the all IoT proposal is the ability to log data, contributing to big analytics potential which can serve different educational purposes. However, privacy and security aspects of the learner should be also actively considered. The system applications must provide a mechanism to treat user anonymously.

\subsection{IoT-Enabled Campus Requirements}

To make use of the new technologies into the educational system, many applications will have to be introduced. These applications will address the needs of the students as well as the teachers and will also serve the institution's administration. These applications can provide the regular e-learning capabilities in a more pervasive nature. The distributed computing power, which is mobile in nature, will have a great effect on the system design and usage. The students' mobile devices will play essential role in applications execution and data access. The widespread use of the mobile smartphone computing is expected to increase the share of the IoT applications including educational applications [9]. 
As a matter of fact, IoT is becoming the future trend of communication and its application affects the way of living and the plethora of smart services it introduces. There are many research areas related to field of IoT communication as it is the major enabler for smart applications and smart cities connectivity infrastructure. IoT requires the development of many smart applications which are built on top of the networking infrastructure in order to achieve the required smart services including eeducations, e-health, e-government, etc. As such, this is a quite interesting area to be explored by comparing the technologies available then making evaluation of several systems that provide communication support to the selected user applications.

According to ITU-T Global Standards, Internet of Things (IoT) is a global infrastructure for the information society, enabling advanced services by interconnecting (physical and virtual) things based on existing and evolving interoperable information and communication technologies [10]. The goal of the Internet of Things is to enable things to be connected anytime, anyplace, with anything and anyone ideally using any network and any service. This goal requires more development in the areas of communications, networking, and applications to become implementable in smart environments.

The push for new IoT technology and applications is growing as more and more people are moving to cities. According to the United Nations, Department of Economic and Social Affairs, more than $65 \%$ of the world's population will be living in urban areas by 2050 [11]. Equivalently, Internet of Everything (IoE) connects people, data, things, and processes in networks of connections [12]. It is viewed as a network of computers and devices of all types and sizes, all communicating and sharing information.

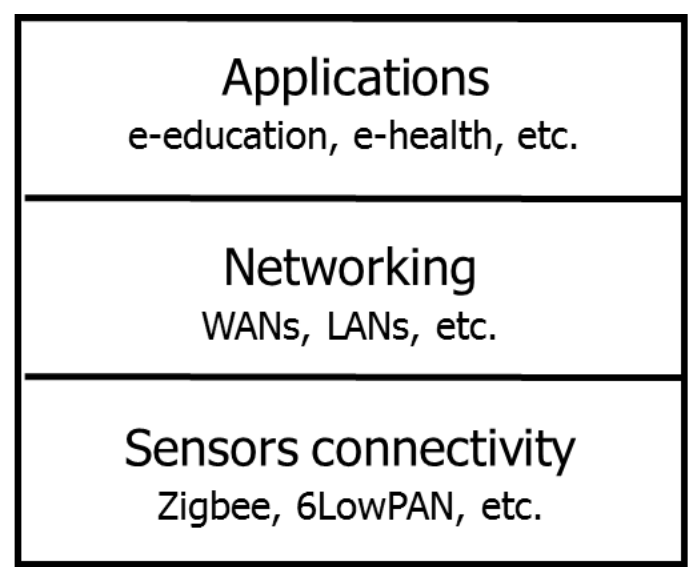

Fig. 1. Overall IOT Architecture

To enable smart educational application, the IoT infrastructure must be put in place. This is to allow smart objects to participate in the educational process. Figure 1 shows the overall IOT architecture and illustrates the relation between the applications and the infrastructure. 
The IoT communication is based on interoperable protocols operating in heterogeneous environments and platforms [13]. Interoperability of different IoT devices must be allowed by the use of different protocols on the sensor layer (Bluetooth, Zigbee, etc.) and different networks connectivity (LANs, WANs).

Cloud Computing is used in order to enable IOT infrastructure. It allows the connection if the billions of devices and sensors that are used in creating the applications. The motivation beyond cloud computing is its ability to be used on demand and as per the requirements of the specific application [14].

\subsection{A Paradigm Shift in the Learning Environment}

There is no doubt of the impact of ICT usage on learning and the learning environment nowadays. The incorporation of such technologies was not necessarily planned but the speed of proliferation in the society has led to adoption in the learning environment. That led to several drawbacks educational institutions suffer from as a result of lack of readiness; take dealing with plagiarism as an example and smart phone use on campuses. With the advent of IoT, it becomes necessary to consider its potential proliferation and adapt correct strategies consequently while investigating its attractiveness from the various perspectives. Those perspectives include, at first hand, the learner who is interested in more adoption of technology with intelligence; the academic staff who are interested in increasing learning effectiveness and reduce administrative efforts; and the administrator who has administration obligations in managing the process, and indeed from the learning process perspective. Assuming the arrival of the IoT to campus entails investigating the different perspectives in needs, readiness, or posing threats.

The Learner Perspective. For learner-centered education, the learner is a first class entity of attention. His preferences, comfort, motivation are important for learning engagement. With the increased use of technology, the younger generations are becoming not only interested in the smart use of technology but they become wellacquainted with its usage. From the student perspective, proper IoT implementations can open the door for higher educational environment usability so as to support the learning results. Students, specially being digital natives can thus perceive the environment as a big controllable digital environment where they can customize their special routes to achieve their educational goals with satisfaction. For the digital native, the more pervasiveness of intelligence in the environment can mean to him/her as paving new faster roads and providing new means of transformation for the nonnative.

Offering flexible learning activities can attract and motivate students especially with social recommendation, not only for self-efficacy but also for motivation support of others. Several services can give the student the comfort and joy of navigating the physical environment such as event recommendation and location, etc. But students with special needs can also benefit from new services through the IoT paradigm. New scenarios of learning support can evolve. There is no doubt of the need to investigate the learner perspective as it is expected that he will be a key player in better control of the environment. 
The Educator Perspective. What are the effects on the educator, and how he/she can benefit from the plethora of intelligence in the environment students are enjoying? Definitely, educators are interested in more students' engagement, better learning effectiveness that the environment is expected to support. Several new automated features can support the teacher. Examples are automated attendance capturing and reporting of learning task completion.

However, it is expected that educators are required to cope with change in the design of learning activities and corresponding academic advising. With the influence of the student crowd as a source of recommendation of activity, it becomes impractical to advise students on different non recommended tasks for example. Furthermore, the design of the learning activities should consider abilities versus preferences based on the student control, to keep the potential of increased usability. In order to maintain a higher usability, the role of the educator becomes to assure the quality of the learning activity but yet to keep as flexibility and control to the learner. Several of the learning activities design can obtain lessons from instructional design in e-learning systems with taking into consideration the cyber-physical systems aspect.

The Learning Administration Perspective. Education governance is expected to experience impacts of such change given the following challenges: create education opportunities, focus on student orientation, enable innovation scenarios, manage user privacy, enable extended times on campus, ensure access equity, manage learning scenarios, and BYOD enablement and strategy.

In order to assess the value of all of the above perspectives, it is necessary to look into the educational scenarios that can come to light with the application of the new technologies based on IoT.

The Educational Scenarios. One student has vision impairment to be able to watch lecture material. His mobile device maintains specific difficulties while he should attend a flexible learning activity. Based on the social recommendation, fellows suggest the learning activity and give him comfort of the ability to finish it. The smart room recognizes different types of difficulties and the IoT devices recommend available accessibility features. The activity facilitator is informed about such difficulties and hence the material is customized to the student; the printer provides the learning material enlarged enough.

The above example shows that introducing IoT-based applications into the educational environment entails the introduction of many new operational scenarios that affects the users. As the majority of the users are students, it is important to understand the needs of the students and their expectations from the new environment. It is equally important to measure the level of knowledge of the students when it comes to the new technology introduction. As such, we conducted a survey on the Computer Engineering students to get their feedback. The survey measures the students' ability to cope with the new technology and their current level of knowledge about IoT in particular. 


\section{Evaluation Experiment: Measuring Knowledge Levels}

\subsection{Experiment Setup}

The following hypotheses are sought for possible verification using the evaluation experiment.

- H1: Students have a fair idea about IoT and some of its aspects and applications

- H2: IoT settings, from the student perspective, add positively to their learning experience

- H3: Students as becoming more digital natives, and with use of technology welcome IoT enablement in the physical learning environments

- H4: A well-defined use case scenario that incorporate IoT adoption is necessary (preferred over just knowledge)

Therefore, the experiment study incorporated the following objectives:

- O1: Evaluate hypotheses

- O2: Measure students' knowledge assessment about IoT

- O3: Obtain students attitudes about IoT in the education environment

- O4: Gain students assessment about the negative sides of IoT

Students from the Department of Computer Engineering at our university participated in the experiment. The participants are from various undergraduate levels. It should be noted that no dedicated courses are provided for IoT related theory and applications currently in our department. Sample questions are provided in Table 1. Questions are evaluated based on Likert scale, [15] where 1 indicates strongly 'Strongly Agree', 2 'Partially Agree', 3 'Neutral or Not Sure', 4 for 'Partially Disagree', and 5 for 'Strongly Disagree'. Consequently, upon calculations, the lower the number the closer it is to a stronger agreement.

Table 1. Sample Experiment Questions in the Likert Scale

\begin{tabular}{|l|l|}
\hline Q & \multicolumn{1}{|c|}{$\begin{array}{c}\text { Likert Question } \\
\text { (1-Strongly agree, 2-Agree, 3-Not Sure, 4-Partially Disagree, 5-Disagree) }\end{array}$} \\
\hline 22 & IoT encourages me to use the latest technology \\
\hline 23 & I prefer enrolling in a campus with IoT technology \\
\hline 24 & IoT connectivity can improve my learning skills \\
\hline 25 & IoT based applications would be more fun to use \\
\hline 26 & I would not use IoT because of privacy concerns \\
\hline 27 & I prefer my mobile be connected to new learning services on campus \\
\hline 28 & I prefer to have the lecture attendance automatically recorded \\
\hline 29 & I believe IoT can help me to learn more technologies \\
\hline 30 & IoT can improve the learning experience for me and my colleagues \\
\hline 31 & I believe we are not yet ready for using IoT now \\
\hline 32 & IoT can have a negative consequence and distractions \\
\hline
\end{tabular}




\subsection{Experiment Results - Evaluation of Hypotheses and Objectives}

Questionnaires are handled and are filled by 25 undergraduate students. Table 2 provides a sample of result summary for some of the questions relevant to stated hypotheses and objectives. Mean, Standard Deviation, and Mode data are provided relevant to each question stated in Table 1.

Table 2. Samples of Question Responses per question

\begin{tabular}{|c|c|c|c|c|c|c|c|c|}
\hline \multirow{2}{*}{ Q } & \multirow{2}{*}{ Mean } & \multirow{2}{*}{ SD } & \multirow{2}{*}{ Mode } & \multicolumn{5}{|c|}{$\begin{array}{c}\text { No. of Participants for each Response } \\
\text { (1-Strongly agree, 2-Agree, 3-Neutral, 4- } \\
\text { Disagree, 5-Strongly Disagree) }\end{array}$} \\
\cline { 6 - 11 } & & & & $(\mathbf{1})$ & $\mathbf{( 2 )}$ & $\mathbf{( 3 )}$ & $\mathbf{( 4 )}$ & $\mathbf{( 5 )}$ \\
\hline 22 & 1.52 & 0.71 & 1 & 15 & 7 & 3 & 0 & 0 \\
\hline 23 & 1.88 & 1.01 & 1 & 11 & 8 & 5 & 0 & 1 \\
\hline 24 & 1.84 & 0.85 & 1 & 11 & 7 & 7 & 0 & 0 \\
\hline 25 & 1.8 & 0.65 & 2 & 8 & 14 & 3 & 0 & 0 \\
\hline 26 & 2.68 & 0.99 & 2 & 3 & 8 & 8 & 6 & 0 \\
\hline 27 & 1.72 & 0.74 & 1 & 11 & 10 & 4 & 0 & 0 \\
\hline 28 & 1.48 & 0.65 & 1 & 15 & 8 & 2 & 0 & 0 \\
\hline 29 & 1.72 & 0.61 & 2 & 9 & 14 & 2 & 0 & 0 \\
\hline 30 & 1.8 & 0.71 & 2 & 9 & 12 & 4 & 0 & 0 \\
\hline 31 & 3.04 & 1.24 & 4 & 4 & 4 & 6 & 9 & 2 \\
\hline 32 & 2.92 & 1.15 & 4 & 4 & 4 & 8 & 8 & 1 \\
\hline
\end{tabular}

\subsection{Analysis and Discussion}

Students have a fair idea about IoT and some of its aspects and applications. Relevant to this hypothesis are 14 survey questions (Q2-Q15) covering the awareness of IoT in general and 5 survey questions (Q16-Q20) covering the awareness of IoT applications. For the first part, it produced (Mean, SD, Mode) as $(2.25,1,2)$ while the second part produces $(1.55,0.67,1)$. The results indicates the application orientation knowledge level of IoT in relation to the comprehensive knowledge for the selected group of Computer Engineering students. This can also serve as input in to evaluate H4. In other words while $62 \%$ either strongly agree or partially agree to awareness of the concept, $90 \%$ do for the application part awareness.

IoT settings, from the student perspective, adds positively to their learning experience. Inputs to this evaluation include Q22, Q23, Q24, Q25, Q27, Q28, Q29, Q30 stated questions in Table 1 and Results for individual questions in Table 2. In general, the results for evaluating this hypothesis can be approximated to $(1.72,0.75$, 1). In general $85 \%$ either agree or partially agree to this hypothesis. The result also coincides with Q30 result, See Table 1, Table 2.

A well-defined use case scenario that incorporate IoT adoption is necessary. Given H1 and the importance of the application domain to be clear to students, Q28 provides a clear scenario and a benefit given to students. The results of this question evaluation is $(1.48,0.65,1)$ which shows clear interest in adopting such scenario. 
$92 \%$ either agree or strongly agree to the question. This also can provide an indicator of digital native's interest in general to adopt technologies for automating tasks that could be more difficult for other generations.

Overall, the survey results shows that the knowledge level of the students is measured to $62 \%$, i.e., not all of them are fully aware of the details on how IoT technology works. This can be attributed to the lack of focus on teaching such newer technologies in the current curriculum. However, $90 \%$ of the students strongly believes that IoT can be used to improve applications in healthcare, transportation, and manufacturing. Most of the students $(80 \%)$ believe that understanding IoT technologies will help them in getting better opportunities in the job market.

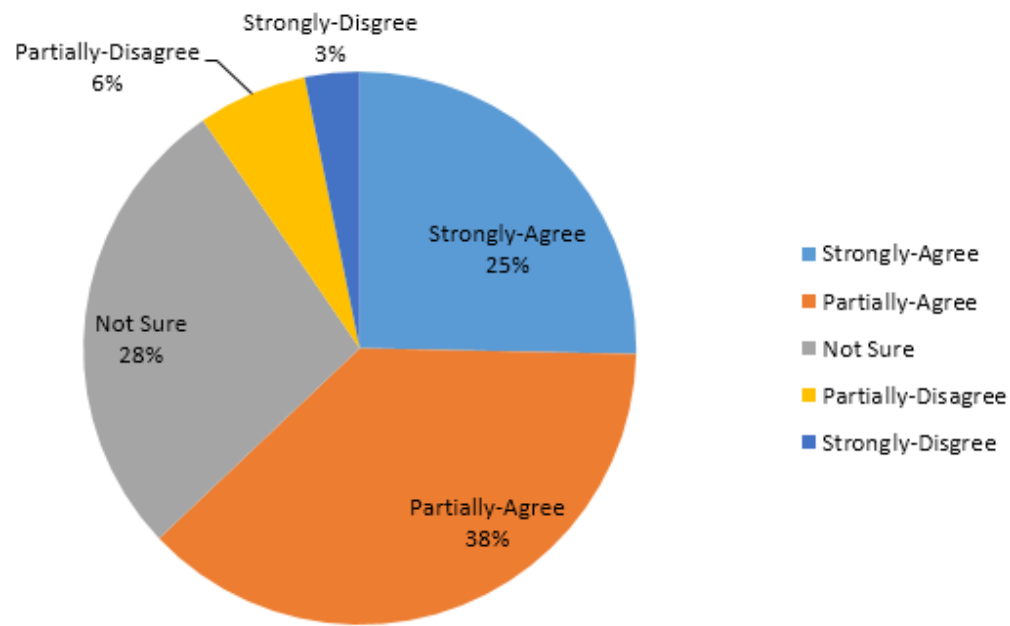

Fig. 2. IoT awareness indicator among experiment participants

A major number of the students $(85 \%)$ indicated that IoT can improve the learning experience for them and their colleagues, and can help them to learn other technologies as well. The students link the learning process to the ability to access all educational services using mobile devices. As a matter of fact, they prefer enrolling in a campus with IoT technology infrastructure. Students, being digital natives (H3), find it natural to use the new IoT technology in the physical learning environments. Figure 2 shows the chart representing the awareness levels of the students regarding IoT applicability.

However, $14 \%$ of the students have negative concerns about IoT in terms of privacy and technology maturity. Issues like location based applications can cause some discomfort as students' privacy is important and good systems should ensure addressing these concerns. Also, some students believe the IoT can have a negative consequence as it can cause more distractions to the learning process and consumes time having to utilize newly introduced services.

Based on student survey results, it becomes clear that the use of IoT infrastructure invites more participation from the students themselves. The student interest in learning and using the latest technologies is a big factor in this equation. 


\section{Conclusion}

It is strongly believed that the area of the IoT can transform the educational environment. While it is expected to have increased connected devices contributing to changes in the daily user behavior, it is important to study and bring this area into education. The work attempts to draw on this importance by visiting relevant research, discuss the different potential and suggest a paradigm with use case scenarios.

The paper shows the importance of considering the different perspectives while focusing on the students perspectives and control over the learning experience. Furthermore, it is expected that the IoT paradigm gives more flexibility on the learning activities and access to learning resources at the hand of the learner. Hence, the learning design should consider the detailing to incorporate the flexible learning experiences, at the activity level, rather than at the course level with well-defined learning and use case scenarios. Consequently, an evaluation study is performed to assess those aspects from the student perspective postulating several hypotheses relevant to student awareness of the technology and its applications, beliefs about its relevant to the learning experience and increased acceptance by digital natives as well as the need to well-defined learning scenarios. The paper discussed the results of the evaluation study which are mainly in support to the hypotheses.

Going forward, with the help of IoT, more information can be collected in order to allow educators and administrators to turn data into actions by providing the university stakeholders with a real-time view of the students, faculty members, and other staff members. This data intelligence enables institutions to make more informed decisions in order to improve student learning experiences, operational efficiency, campus security, and many other aspects of the educational environment promoting more creativity.

\section{Acknowledgment}

Appreciation goes to anonymous student participants from Computer Engineering Department for contributing to the evaluation experiment.

\section{References}

[1] Atzori, L., Iera, A., and Morabito, G. (2010). The Internet of Things: A survey, Computer Networks, vol. 54, Issue 15, 28 October 2010, pp 2787-2805. https://doi.org/10.1016/j.com net.2010.05.010

[2] Pal, A., Mukherjee, A., and Dey, S. (2016). Future of Healthcare-Sensor Data-Driven Prognosis, Springer Series in Wireless Technology, International Publishing Switzerland, https://doi.org/10.1007/978-3-319-42141-4 9

[3] Soliman, M. and Elsaadany, A. (2016). Smart Immersive Education for Smart Cities with Support via Intelligent Pedagogical Agents, Proceedings of the 39th International Convention on Information, Communication Technology, Electronics and Microelectronics. https://doi.org/10.1109/MIPRO.2016.7522247 
Paper-Experimental Evaluation of Internet of Things in the Educational Environment

[4] Prensky, M. (2001). Digital Natives, Digital Immigrants. On the Horizon, 9(5) Retrieved from http://www.marcprensky.com/writing/Prensky\%20-\%20Digital\%20Natives,\%20Digi tal\%20Immigrants\%20-\%20Part1.pdf, Last accessed May 10, 2017. https://doi.org/10.1108/10748120110424816

[5] Chandrahasan, M. et. al. (2016). Survey on Different Smart Parking Techniques, International Journal of Computer Applications (0975 - 8887), vol. 137, no.13.

[6] Elsaadany, A. (2015). Campus Crowd Sensing Platform using Mobile Computing for Smart Education Environments, Proceedings of Fifth International Conference on Information Communications Technology, Alexandria.

[7] Atzori, L. et. al. (2012). The Social Internet of Things (SIoT) - When social networks meet the Internet of Things: Concept, architecture and network characterization, Computer Networks, Volume 56, Issue 16, 14 November 2012, Pages 3594-3608 https://doi.org/10.1016/j.comnet.2012.07.010

[8] Mashal, O. A. and Chung, T.Y. (2016). Analysis of recommendation algorithms for Internet of Things, IEEE Wireless Communications and Networking Conference Workshops (WCNCW), pp. 181-186.

[9] Kamilaris, A. and Pitsillides, A. (2016). Mobile Phone Computing and the Internet of Things: A Survey, IEEE Internet of Things Journal.

[10] ITU-T Global Standards Initiatives Recommendation ITU-T Y.2060 (06/2012) http://www.itu.int/en/ITU-T/gsi/iot/Pages/default.aspx, Last accessed May 2016

[11] United Nations, Department of Economic and Social Affairs, Population Division, World Urbanization Prospects, 2014.

[12] Evans, D. (2011). The Internet of Things How the Next Evolution of the Internet Is Changing Everything, Cisco IBSG.

[13] Vermesan, O. and Friess, P. (2013). Internet of Things: Converging Technologies for Smart Environments and Integrated Ecosystems, River Publishers Series in Communications.

[14] Buyya, R. and Dastjerdi, A. (2016). Internet of Things Principles and Paradigms, 1st Edition.

[15] Likert, R. (1932). A technique for the measurement of attitudes. Archives of Psychology. vol. 22 , no. 140 , pp. $1-55,1932$

\section{$7 \quad$ Authors}

Amr Elsaadany is an Assistant Professor at the Department of Computer Engineering. He received M.Sc. and Ph.D. in Computer and Information Science at the Ohio State University. His research interests are Networking Protocols, Internet of Things, Mobile Computing, and Participatory Sensing.

Mohamed Soliman is an Assistant Professor at the Department of Computer Engineering. He received $\mathrm{PhD}$ in Computer Science from Graz University of Technology, Austria and M.Sc. in Computer Science from the University of Waterloo, Canada. His main research interests are in the area of technology enhanced learning. 